\title{
Synchronization of JESD204B-based ADCs
}

\author{
Tao Yan ${ }^{1}$,Shanqing $\mathrm{Hu}^{2^{*}}$,Xingming $\mathrm{Li}^{3}$ \\ ${ }^{1}$ Beijing Key Laboratory of Embedded Real-timeInformation Processing Technology, Beijing Institute of \\ Technology, Beijing, 100081, China \\ ${ }^{2}$ Beijing Key Laboratory of Embedded Real-timeInformation Processing Technology, Beijing Institute of \\ Technology, Beijing, 100081, China \\ ${ }^{3}$ Tsinghua University, Beijing, 100081, China \\ yantao93@live.com ${ }^{1}$,hushanqing@bit.edu.cn²,1xm11544@163.com³
}

Keywords: JESD204B, deterministic latency, synchronization, Radar system

\begin{abstract}
With the rapid development of radarsignal processing systems, a great demand of high-speed communication is required. JESD204B interface has become an outstanding solution for ADC-related data transmission, and has been widely applied. However, the synchronization of multiply JESD204B-based ADCs has been a challenge because of the special feature such as deterministic latency. In this paper, we design a data acquisition system integrated two JESD204B-based ADCs, and proposed a general and flexible synchronization method based on FPGA, by using and controlling deterministic latency. As is validated by the practical measurements, the method can maintainaphase difference between the two 2.5Gbps ADCsfrom $17 \mathrm{ps}$ to 22 psstably, forthe signals of frequencies less than $1.8 \mathrm{GHz}$.
\end{abstract}

\section{Introduction}

Conventionally, CMOS or LVDS is employedto transmit the acquired data between ADC with the processing unit, such as FPGA, but recently the JESD204B interface has change this situation, and become more and more accepted. Rather than CMOS and LVDS, the JESD204B interface offers several advantagesin terms of speed,size, and cost. Its data transfer rate can reach up to $12.5 \mathrm{Gbps}$, and this provides sufficient bandwidth to keep balance with the ever increasingADC sampling rate[1].

In high-complexity signal processing system designs, multiple analog-to-digital converters(ADC) are commonly integrated to obtain larger dynamic ranges and high performance, and their synchronization determines the overall performance. As for theJESD204Binterface, it introduces a deterministic latency for each converter in the system, and this helps to make multi-ADCs synchronized.

In previous work, multiple ADCs of JESD204B interface wasused indownlink data reception and processing of the digital phased-array radar, and the two ADCs inside the AD9250 wassynchronized by using deterministic latency[2]. In another paper [3], the two ADCs inside the JESD204B-based AD9680 was also implemented by using deterministic latency, and thephase differencewas limited to $45 \mathrm{ps}$. The previous work makes it available for synchronizing two ADCs inside a chip, but the synchronization becomes more challenging when it comes to independent ADCs.

In this paper, we design data acquisition systemby integrating twoindependentAD9625 provided by Analog Devices,Inc.(ADI) company, which is a 12-bitconverter and compatiblewith JESD204B. As the other termination, we use a XilinxVertx-7FPGA as the controller and receiver, whose development kit is Vivado2015.4. By using and controllingthe deterministic latency, we propose a generaland flexible synchronization method based on FPGA. The method is validated bythe practical measurements, and it can control the phase difference between the two AD9625 less than $20 \mathrm{ps}$ for various frequencies, under2.5Gsps condition. 
The rest paper is arranged as follows. The deterministic latency of the JESD204B is introduced in section II. Our method for synchronizing multiple ADCs is described in section III. The section IV concludes the paper.

\section{Deterministic Latency of JESD204B}

Deterministic latency across the JESD204B link is defined by the time it takes serial data to propagate from the parallel framed data input at the transmitter to the parallel de-framed data output at the receiver.As illustrated in Fig.1, it includes three components: the delay from the transmitter to the output, the spatial routing delay, and the receiver delay from the input to the de-framer. Different ADCs have their own unique deterministic latency, even in the same system.

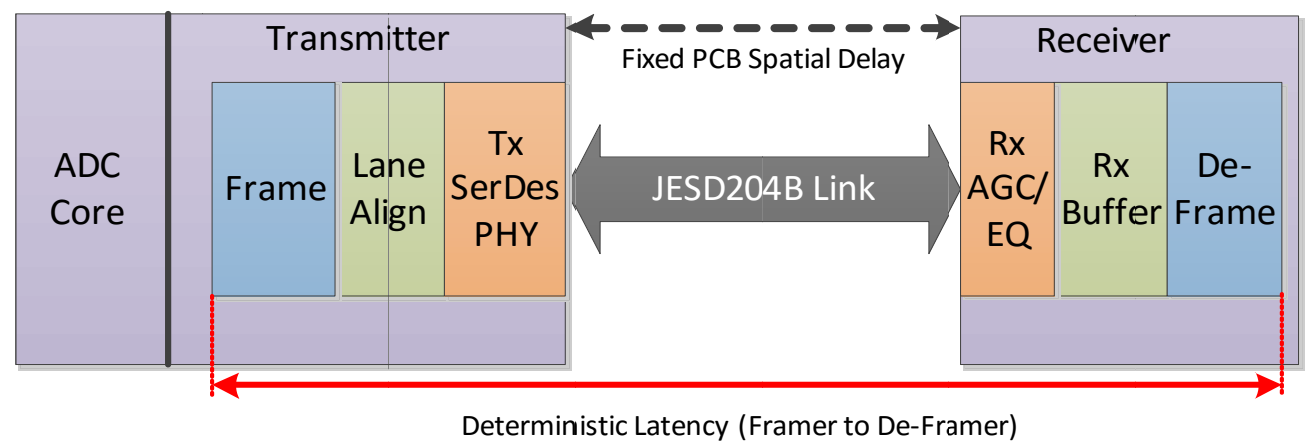

Fig.1 Illustration of Deterministic Latency

There are three subclasses of the JESD204B protocol, and they have different deterministic latencydefinition. Here we chose the paper-used subclass 1 for further demonstration.

The Principle of Deterministic Latency for Subclass 1. Subclass 1 supports deterministic latency by usinga SYSREF signal as the reference. As illustrated in Fig.2, the frameclockand multi-frameclock are aligned internally to theSYSREF edge.

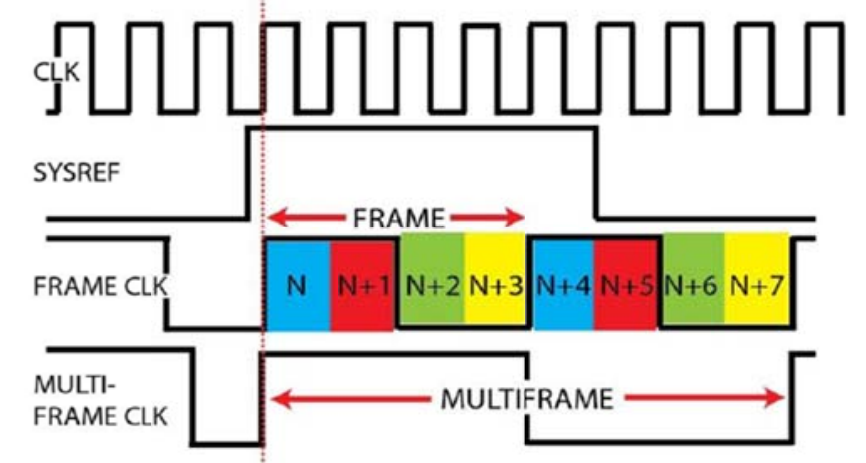

Fig.2 Samples are aligned after the clock latches a SYSREF edge

Subclass 1 defines a receiver buffer, and its release time is referenced by theSYSREF signal. The bufferenables the receiver to deskew the samples of early arrival of data to match with the latest arrival of databy using buffer delay [4], as illustrated in Fig.3.As a result, the multi-frames of different links are aligned to the multi-frame clock by using buffer delaywithin the receiver.
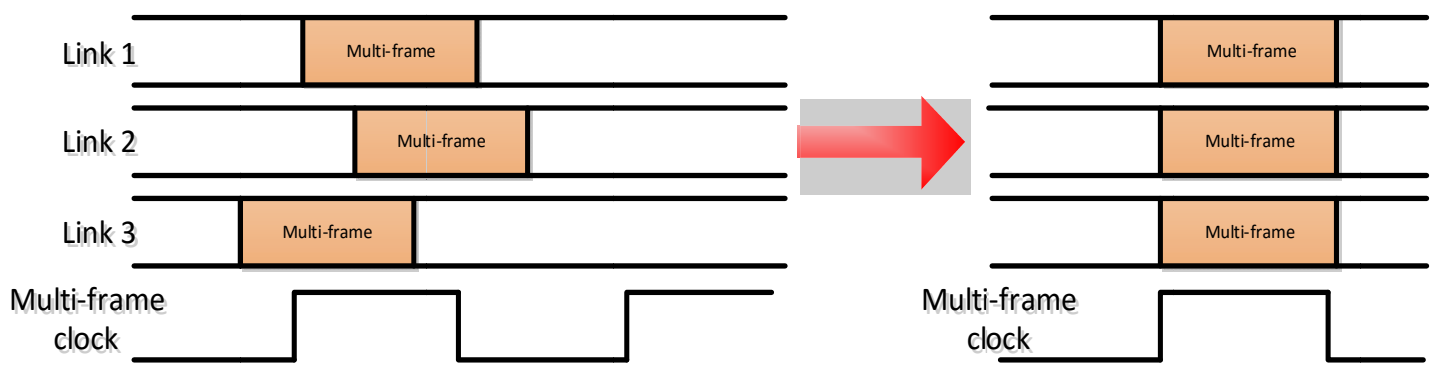

Fig.3The function of the receiver buffer 
Here the period of aforementioned SYSREF must be multiple of the period oflocal multi-frame clock (LMFC), and it shouldbe synchronous with the device clock and be accurately controlled to make sure afixed clock edge.

The Implementation of Deterministic Latency for subclass 1.Although the deterministic latency offers a good way for synchronization, the deterministic latency uncertainty (DLU) limits its implement, and need some compensatations. The DLU means the LMFC skew and itincludes two parts: the first is theun-controlled distribution skewof the device clocks (DCLK) in the system, and the other is SYSREF distribution skew (DS ${ }_{\text {SYSREF }}$ ), which means thetime difference between the earliest arriving SYSREF and the last one.

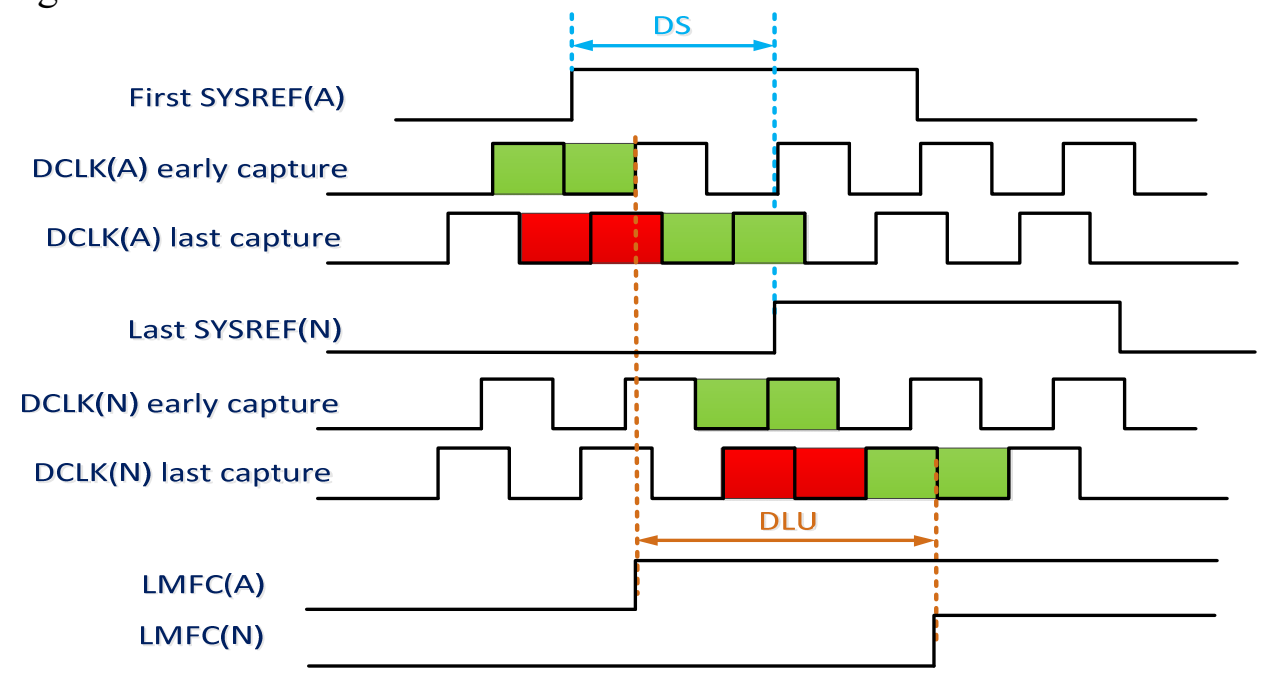

Fig.4Deterministic Latency Uncertainty

Since the JESD204B-basedADCs usually workat a very high rate, itssetup and hold time requirements become challenging [5].To meet this, each device in the JESD204B system should have its own SYSREF/DCLK pair, and their phase delay should be adjusted to ensure the timing [6], as illustrated in Fig 5.The SYSREF can be delayed in fixed increments, and a phase that makes the SYSREF edge near to middle of the valid window is recommended to ensure the minimum DLU.

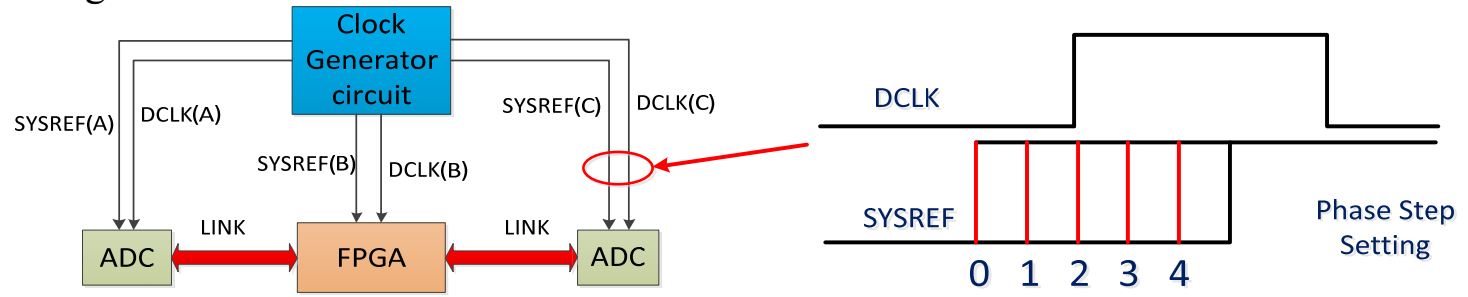

Fig.5 Adjustment of the SYSREF phase delay tominimum DLU

\section{Design of JESD204B-based ADCs Synchronization}

With thedemand of data acquisition precision and high sampling rate, the data acquisition system integratingFPGA and ADC has become a mainstream of the industry design. In the section, we elaborate our data acquisition system with FPGAs and ADCs integrated, and demonstrate our method for synchronizing multiply JESD204B-based ADCs.

Demonstrationof theData AcquisitionSystem. The data acquisition system is comprised of mother and daughter boards for versatility and flexibility considerations. The mother board implements controlling, processing, and data handing out, andthe daughter board acquires and transfers external signals. They are connected through the high-pin count (HPC) connector (400 pins), whichsupportsup to $10 \mathrm{Gbps}$ per lane.

As illustrated in Fig.6 (left), the Xilinx Virtex-7 in the mother board communicateswith daughter board including the JESD204B interface through two HPC connectors. The daughter board includes a clock driver AD9520from ADI and a convert AD9625. The former offers clock for the latter, and 
the latter communicates with Virtex-7 FPGA through JESD204B interface, as illustrated in Fig.6 (right). The AD9625 is a 12-bit monolithic ADC that can operate at conversion rate up to 2.5Gsps.

HPC

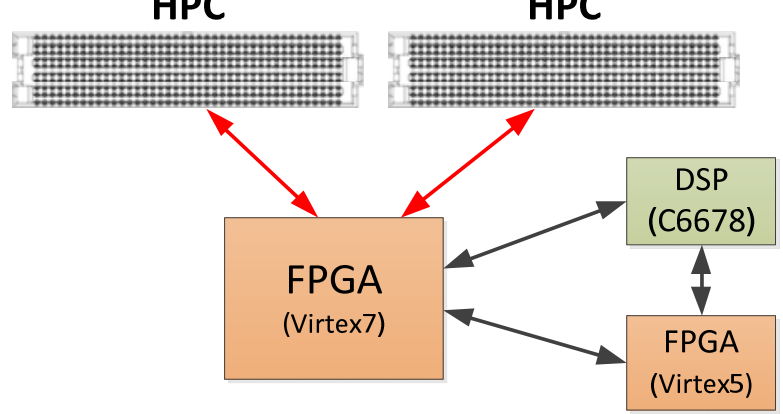

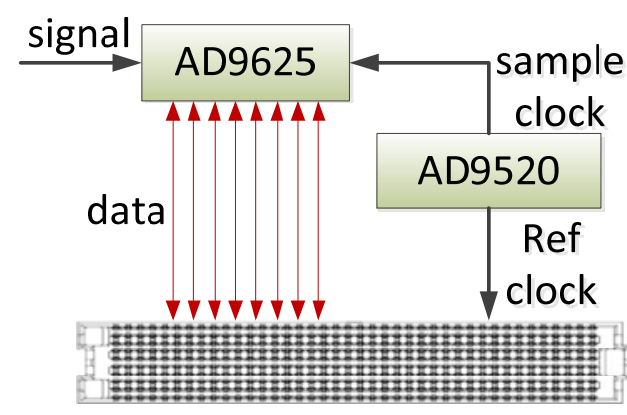

Fig.6 Design of the mother board and the daughter board

Design and Implement of the Synchronization based on FPGA.We design aJESD204B controller based on FPGA, and it includes three modules: high-speed serial receiving module, deterministic latency signalgeneration module, and the configuration module, as is illustrated in Fig.7.The high-speed serial receiving module receives data through Gigabit Transceiver(GTX), the deterministic latency signal generation module is used to generate the SYSREF signal, and the configuration module configures the setup parameters to AD9520, AD9625, and FPGA GTX core.

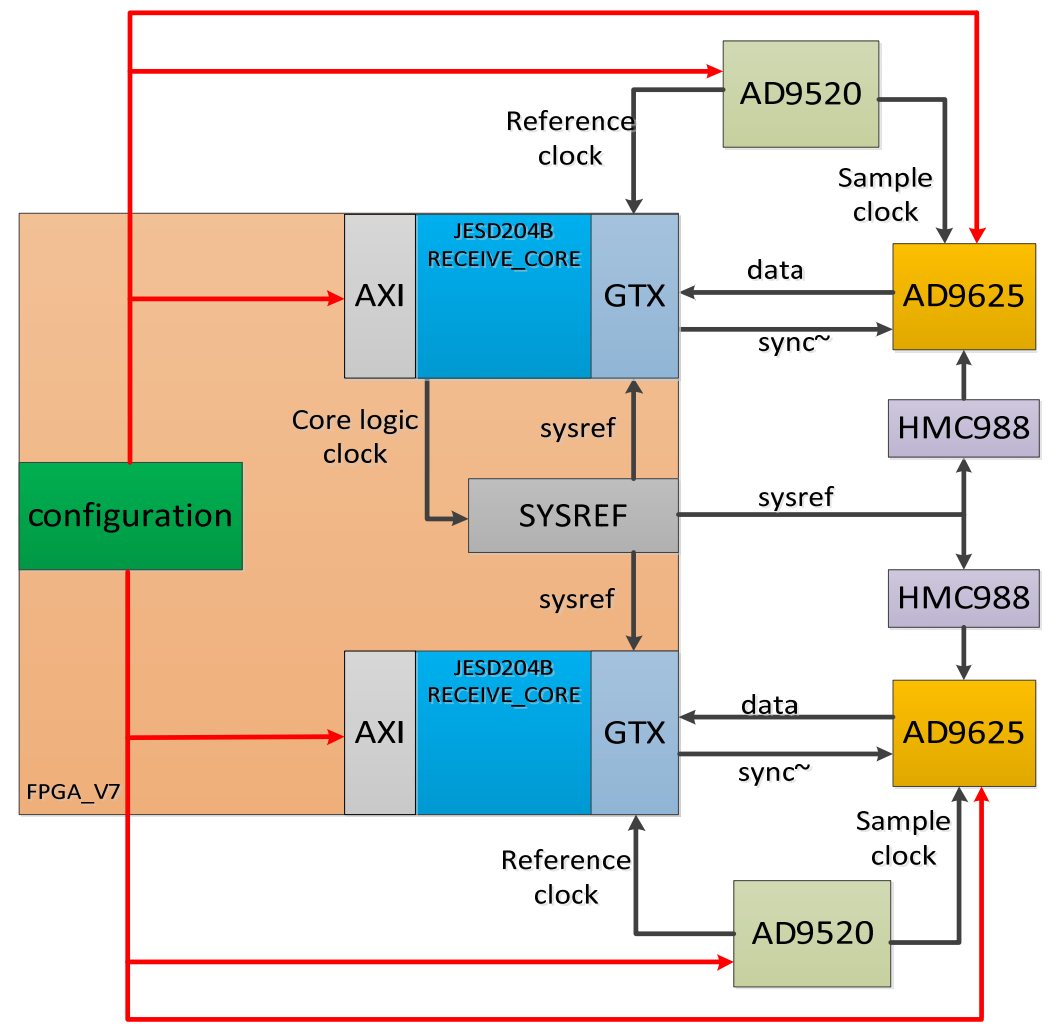

Fig.7 Implementation ofmultipleADCs synchronized samplingbased on FPGA

Weuse 8 lanes in our design, and we determined other parameters, according to the specificationof AD9625[7]. Bysetting the ADC sampling rate as maximum 2.5Gsps, we can calculate the multi-frame clock as $19.53125 \mathrm{MHz}$, and here we set the SYSREF frequency equal tothe multi-frame clock as $19.53125 \mathrm{MHz}$. To minimizethe DLU,we adjust the SYSREF phase delay by HMC988, which is capable of adjusting SYSREF phase delay in 20ps steps.

The result of multiple synchronized ADCs based on JESD204B. Thetest setup of multiple synchronized ADCs is shown in Fig 8. A signal generator is used to provide sampled signal of $12 \mathrm{dBm}$ and the other providesa $10 \mathrm{MHz}$ reference clockof $10 \mathrm{dBm}$. To eliminate the influence of transmission between the two daughter boards, the length of transmission lane are kept equal and 
the signal should divide by a power divider.To ensure the accuracy and robustness of the achievedphase difference of the two ADCs, we testedseveral incremental frequenciesfrom $10 \mathrm{MHz}$ to $1800 \mathrm{MHz}$, when the AD9625 working in $2.5 \mathrm{Gsps}$.

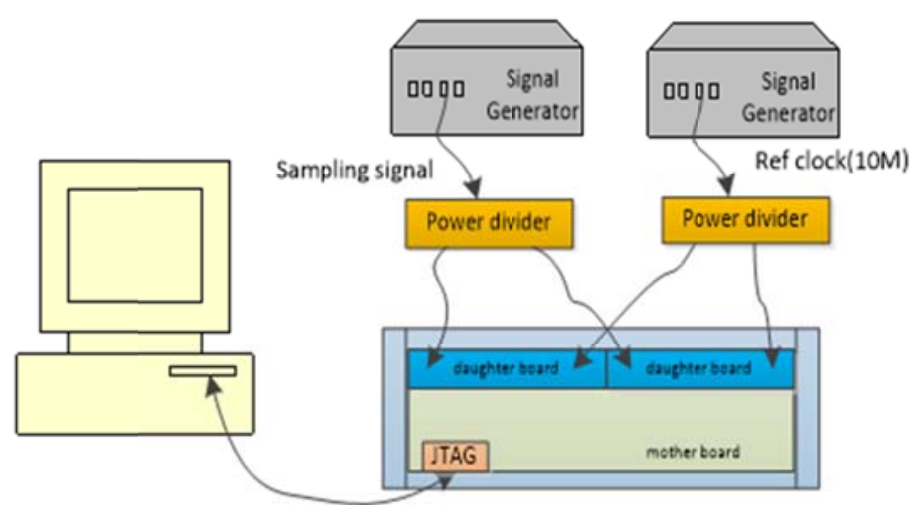

Fig.8 Test of multiple synchronized ADCs

Using FFT to calculate the imaginary part and the real part of the signal, the signal phase is the arctangent of the ratio of the imaginary part and the real part. We analyze the data by Matlab andthe result of phase difference value between the two ADCs is shown in Fig.9.

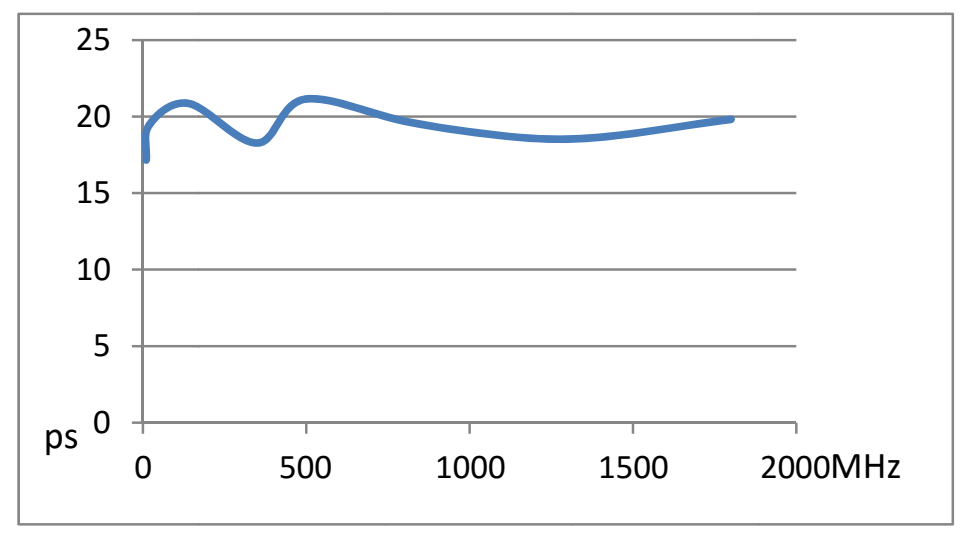

Fig.9the phase difference value between the two ADCs

\section{Conclusion}

To satisfy the demand ofhigh sampling rate and high transmission rate, we design a data acquisition system integrated two JESD204B-based ADCs. To implement the synchronization of the ADCs, we proposed a general and flexible synchronization method based on FPGA, by using and controlling deterministic latency. Through the measurements and data analysis, it isconclude that the phase difference value between the two $2.5 \mathrm{Gsps}$ ADCs is well controlled from $17 \mathrm{ps}$ to $22 \mathrm{ps}$ stably, for the signals of frequencies less than $1.8 \mathrm{GHz}$. The result shows that the design of multiple ADCs synchronized has a high performance for Radar systems.

\section{Acknowledgement}

This workwas supported by the Chang Jiang Scholars Programme (Grant No.T2012122) and the Hundred Leading Talent Project of Beijing Science and Technology (Grant No. Z141101001514005).

\section{References}

[1] “JESD204B Survival Guide: Practical JESD204B Technicallnformation,Tips,and Advice from the World's Data Converter MarketShare Leader",Analog Devices Inc.2013 
[2] Chen Yang, Yu Yuxin, Xi Jun. Application of downlink synchronization acquisition technology for phase-array radar based on JESD204B protocol [J]. RADAR \& ECM, 2015.6.

[3] Xiao Dandan.Design and Verification of Wideb and High-dynamic Full Digital Radar Receiver Based on AD9680 [J]. Electronic Sci. \& Tech,october.15, 2015.

[4] Lan Beavers. Demystifying Deterministic Latency Within JESD204B Converters. Analog Devices Inc. MS-2626

[5] Ian Beavers. Synchronize Multiple ADCs with JESD204B [J].Electronic design, 2013, 61(12):16-17.

[6] Matt Guibord. JESD204B multi-device synchronization: Breaking down the requirements[J].Analog Applications Journal.2015.

[7] AD9625 [M].USA: ADI, 2014 